\title{
CÂNCER DO ESÔFAGO EM PACIENTE COM MEGAESÔFAGO CHAGÁSICO
}

\author{
Maria Aparecida Coelho de Arruda HENRY, Mauro Masson LERCO e Walmar Kerche de OLIVEIRA
}

RESUMO - Racional - O megaesôfago constitui problema de saúde pública em nosso país, pois acomete indivíduos em sua fase de maior produtividade. Os doentes com essa afecção podem apresentar em sua evolução associação com câncer do esôfago. Objetivo - Analisar os aspectos clínicos e epidemiológicos de pacientes com megaesôfago e câncer do esôfago. Métodos - Foram avaliados de maneira retrospectiva 20 pacientes com megaesôfago e câncer (grupo 1) e 20 com câncer do esôfago (grupo 2). Estudaram-se os dados demográficos, hábitos (etilismo e tabagismo), tipo histológico do tumor, localização da lesão, diferenciação celular, estádio, tratamento e sobrevida. Resultados Não foi observada diferença entre os grupos, com relação à idade, sexo, localização da lesão, tipo histológico do tumor, diferenciação celular, estádio e sobrevida. Com relação aos hábitos de vida, a associação de etilismo e tabagismo foi observada em maior número de pacientes com câncer do esôfago sem o antecedente de megaesôfago. Conclusões - As características clínicas dos pacientes com megaesôfago e câncer não diferem daqueles com neoplasia maligna esofágica não associada ao megaesôfago, principalmente no que se refere ao prognóstico desfavorável frente ao tratamento instituído. Nos pacientes com megaesôfago o tumor pode se localizar em qualquer porção do órgão.

DESCRITORES - Acalásia esofágica. Neoplasias esofágicas. Seguimentos.

\section{INTRODUÇÃO}

O câncer de esôfago constitui a oitava neoplasia, em termos de incidência mundial. O diagnóstico desta doença, é realizado na maioria dos casos em fase avançada, razão pela qual a afecção está associada a altas taxas de morbidade e mortalidade ${ }^{(34)}$.

O tumor maligno do esôfago ocorre com maior freqüência em indivíduos de idade avançada e do sexo masculino ${ }^{(9)}$, em geral associado a fatores como etilismo e tabagismo ${ }^{(11)}$, ingestão de alimentos ou bebidas quentes $^{(1,18,23,25,32)}$, vírus, deficiências vitamínicas ${ }^{(27)}$; higiene oral deficiente, ingestão de substâncias cáusticas ${ }^{(22,31)}$, divertículo de Zenker ${ }^{(24)}$ e megaesôfago ${ }^{(2)}$.

O tipo histológico mais freqüente é o carcinoma espinocelular. Nas últimas décadas a incidência de adenocarcinoma do esôfago tem aumentado, associada à metaplasia epitelial, complicação grave da doença do refluxo gastroesofágico ${ }^{(16,29)}$.

A prevalência do câncer do esôfago em pacientes com megaesôfago chagásico é variável. BRANDALISE et al. ${ }^{(3)}$ analisaram a evolução de 140 pacientes com megaesôfago e verificaram que $9,2 \%$ destes desenvolveram câncer do esôfago. Estudo semelhante conduzido na Faculdade de Medicina da Universidade de São Paulo, SP, por PINOTTI et al. ${ }^{(21)}$, mostrou a ocorrência de 12 casos de câncer de esôfago em 308 portadores de megaesôfago (3,9\%).

O objetivo deste trabalho foi analisar os aspectos clínicos e epidemiológicos de pacientes com a associação megaesôfago e câncer do esôfago.

\section{MÉTODOS}

Foram estudados de maneira retrospectiva, 40 pacientes com câncer do esôfago atendidos no período de 1993 a 2003. Esta casuística é constituída de 36 homens e 4 mulheres, com idades variando entre 35 e 72 anos (média de 55 anos). Os pacientes foram divididos em 2 grupos de 20:

- grupo 1: pacientes com câncer do esôfago e megaesôfago

- grupo 2: pacientes com câncer do esôfago

Os pacientes de ambos os grupos apresentavam queixa de disfagia e foram submetidos a exames radiológico e endoscópico de esôfago, tomografia computadorizada de tórax e abdome e broncoscopia. Aqueles do grupo 1 referiam antecedentes epidemiológicos para doença de Chagas, confirmada através da reação sorológica de Machado Guerreiro.

A análise dos prontuários permitiu a obtenção de dados referentes a idade, sexo, hábitos (etilismo e tabagismo), tipo histológico do tumor, localização da lesão, diferenciação celular, estádio, tratamento e sobrevida.

Dos 20 pacientes do grupo 1, 6 apresentavam associação com megacólon, 1 com cardiopatia e 4 já haviam sido operados, visando o tratamento do megaesôfago.

Visando a homogeneidade das amostras, para o grupo 2 foram selecionados 20 pacientes com câncer do esôfago atendidos no mesmo período e com as mesmas características demográficas, tipo histológico do

Departamento de Cirurgia e Ortopedia - Faculdade de Medicina de Botucatu, Universidade Estadual Paulista, Botucatu, SP.

Correspondência: Dra. Maria Aparecida Coelho A. Henry - Rua Miguel Cioffi, 200 - 18607-790 - Botucatu, SP. E-mail: rhenry @ibb.unesp.br 
tumor, estádio dos pacientes do grupo 1 e sobrevida informada pelos familiares.

Os dados foram submetidos a análise estatística ${ }^{(19)}$ (teste exato de Fisher e análise de variância). O nível de significância utilizado foi de $5 \%$.

\section{RESULTADOS}

\section{a) Distribuição dos pacientes por sexo e idade}

A distribuição dos pacientes por sexo não mostrou diferença na comparação entre os grupos, pois havia 18 homens e 2 mulheres em cada casuística estudada. A idade média dos pacientes com megaesôfago e câncer (grupo 1) foi de 56,1 1 8,8 anos e naqueles do grupo 2 , de 54,7 $\pm 9,8$ anos. A análise estatística não mostrou diferença significante entre os grupos $(P=0,64)$.

\section{b) Localização do tumor}

Nos pacientes com a associação megaesôfago e câncer, a distribuição das lesões foi homogênea, pois em 8 doentes o tumor estava situado no terço superior, 8 no terço médio e 4 no inferior. Naqueles com câncer do esôfago (grupo 2), as lesões predominaram no terço médio. Todavia esta diferença não mostrou significância estatística $(P=0,08)$ (Tabela 1$)$.

\section{c) Hábitos}

Dos 40 pacientes estudados, 29 referiam a ingestão de bebidas alcoólicas em altas doses e por tempo prolongado, associada ao tabagismo. Essa associação foi observada em 10 pacientes do grupo 1 e em 19 do grupo 2. Os três pacientes que negavam os hábitos de etilismo e tabagismo tinham megaesôfago chagásico (Tabela 2).

\section{d) Tipo histológico e diferenciação celular do tumor}

O exame anatomopatológico das lesões demonstrou a presença de carcinoma espinocelular nos 40 pacientes desta casuística. A distribuição dos pacientes quanto a diferenciação celular dos tumores encontra-se na Tabela 3.

\section{e) Estádio do tumor e do megaesôfago}

O estádio do tumor foi efetuado após análise dos exames radiológicos e endoscópicos, que evidenciavam a extensão da lesão, invasão de órgãos contíguos (traquéia, brônquios) ou metástases à distância (pulmonar ou hepática). Em 18 doentes do grupo 1 e em 19 do grupo 2 o tumor encontrava-se nos estádios III e IV (classificação TNM da União Internacional Contra o Câncer - UICC 1987).

O estádio do megaesôfago, baseado na avaliação dos esofagogramas, permite observar que em $21 \%$ dos casos os pacientes encontravamse em fase incipiente (grau I), em 47,4\% fase não-avançada (graus II e III) e 31,6\%, fase avançada (grau IV) ${ }^{(26)}$.

\section{f) Sobrevida}

A sobrevida dos pacientes do grupo 1 foi de $8 \pm 8,8$ meses, naqueles do grupo 2 de $5,8 \pm 5$ meses $(P=0,34)$.

A sobrevida dos pacientes segundo o grau da diferenciação celular, não mostrou diferença significante (Tabela 4).

\section{g) Tratamento}

O tratamento cirúrgico paliativo foi a opção terapêutica adotada na maioria dos pacientes. Em apenas um paciente do grupo 1 foi realizada a ressecção do esôfago e esofagogastroplastia (Figuras 1 e 2,
TABELA 1. Câncer do esôfago em pacientes com (G1) e sem (G2) megaesôfago chagásico. Distribuição segundo a localização do tumor

\begin{tabular}{lcccc}
\hline Grupo & $1 / 3$ superior & $1 / 3$ médio & $1 / 3$ inferior & Total \\
\hline 1 & 8 & 8 & 4 & 20 \\
2 & 3 & 15 & 2 & 20 \\
Total & 11 & 23 & 6 & 40 \\
\hline$P=0,08$ & & &
\end{tabular}

TABELA 2. Câncer do esôfago em pacientes com (G1) e sem (G2) megaesôfago chagásico. Distribuição segundo os hábitos de vida

\begin{tabular}{lccccc}
\hline Grupo & Etilismo + tabagismo & Etilismo & Tabagismo & Abstêmio & Total \\
\hline 1 & 10 & 4 & 3 & 3 & 20 \\
2 & 19 & 0 & 1 & 0 & 20 \\
Total & 29 & 4 & 4 & 3 & 40 \\
\hline $\begin{array}{l}P=0,006 \\
\text { Teste exato de Fisher }\end{array}$ & & & & &
\end{tabular}

TABELA 3. Câncer do esôfago em pacientes com (G1) e sem (G2) megaesôfago chagásico. Distribuição segundo a diferenciação celular

\begin{tabular}{lcccc}
\hline Grupo & Pouco diferenciado & $\begin{array}{c}\text { Moderadamente } \\
\text { diferenciado }\end{array}$ & Bem diferenciado & Total \\
\hline 1 & 2 & 11 & 7 & 20 \\
2 & 5 & 11 & 4 & 20 \\
Total & 7 & 22 & 11 & 40 \\
\hline
\end{tabular}

$P=0,35$

Teste exato de Fisher

TABELA 4. Média e desvio-padrão referentes a sobrevida (meses) nos grupos 1 e 2, segundo a diferenciação celular

\begin{tabular}{lcccc}
\hline Grupo & Pouco diferenciado & $\begin{array}{c}\text { Moderadamente } \\
\text { diferenciado }\end{array}$ & Bem diferenciado & $\boldsymbol{P}$ \\
\hline 1 & $3,0 \pm 4,2$ & $10,5 \pm 10,9$ & $5,2 \pm 3,8$ & 0,36 \\
2 & $5,7 \pm 5,0$ & $6,2 \pm 4,9$ & $4,9 \pm 6,8$ & 0,91 \\
\hline \multicolumn{2}{l}{ Análise de variância } & & &
\end{tabular}

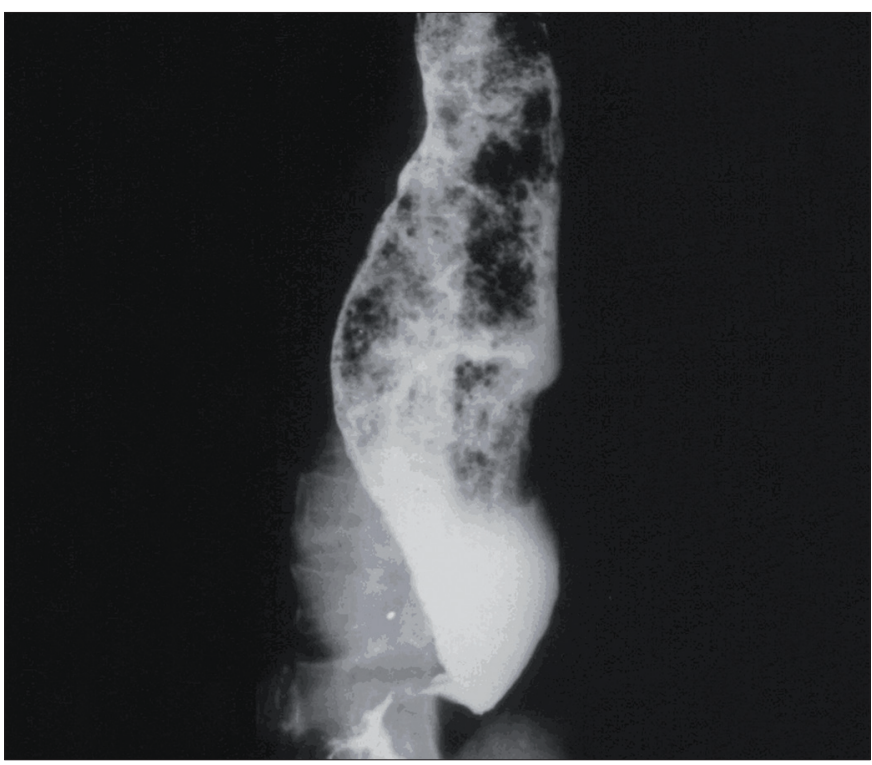

FIGURA 1. Esofagograma demonstrando imagem de subtração em pacientes com megaesôfago chagásico 
Tabela 5). O tratamento radioterápico foi realizado em 9 pacientes do grupo 1 e em 10 do grupo 2, tendo em vista a irresecabilidade da lesão.

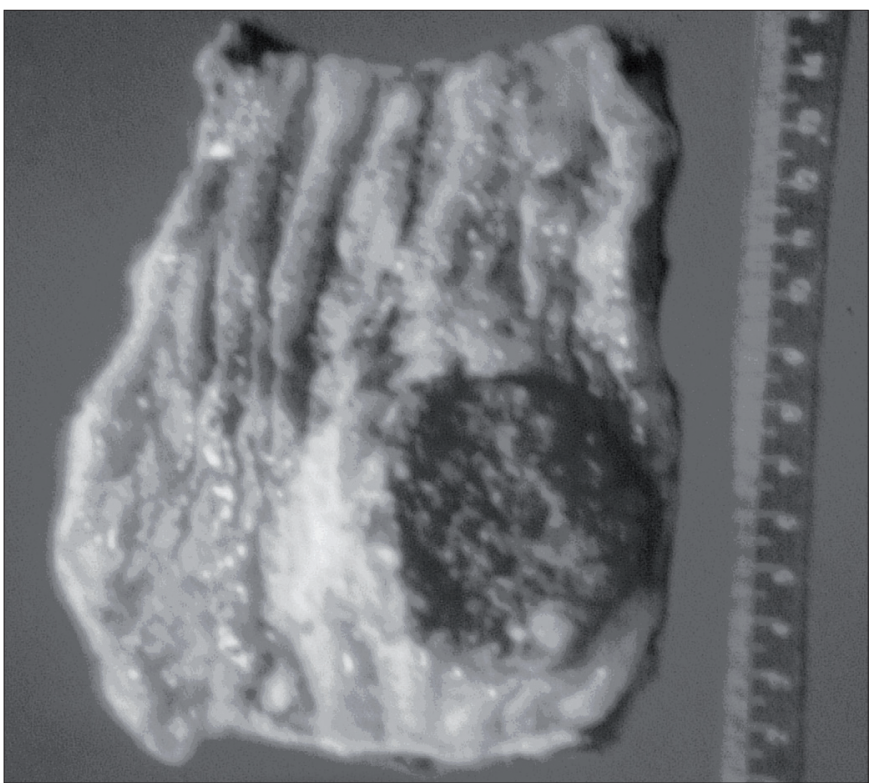

FIGURA 2. Produto de esofagectomia subtotal no paciente com megaesôfago chagásico (grupo 1)

TABELA 5. Câncer do esôfago em pacientes com (G1) e sem (G2) megaesôfago chagásico. Tratamento instituído

\begin{tabular}{lccccc}
\hline Grupo & Esofagectomia & Gastrostomia & Jejunostomia & Não operado & Total \\
\hline 1 & 1 & 6 & 4 & 9 & 20 \\
2 & 0 & 10 & 10 & 0 & 00 \\
Total & 1 & 16 & 14 & 9 & 40 \\
\hline
\end{tabular}

$P=0,002$

Teste exato de Fisher

\section{DISCUSSÃO}

Após a descrição da associação megaesôfago e câncer por FAGGE (1872) citado por CÂMARA-LOPES(5), vários trabalhos foram publicados, confirmando o achado desse autor ${ }^{(2,3,4,5,12,17)}$.

Em levantamento sobre o assunto em questão, encontrou-se apenas um estudo onde os autores não reconhecem como válida a associação entre megaesôfago e câncer ${ }^{(6)}$. Essa assertiva baseia-se em estudo retrospectivo de 153 doentes com câncer do esôfago atendidos em período de 11 anos, no qual não se observou nenhum caso de megaesôfago. Todavia, na presente série, o resultado foi diferente, pois a análise dos protocolos de 165 pacientes com câncer de esôfago e seguidos em igual período mostrou a existência de 20 doentes com megaesôfago (grupo 1).

A prevalência de câncer do esôfago em pacientes com megaesôfago no serviço onde o presente estudo foi desenvolvido, é de $10 \%$. Este resultado é semelhante ao observado por BRANDALISE et al. ${ }^{(3)}$, porém superior ao relatado por outros autores $^{(4,5,21,30)}$.
Quanto à localização do tumor, a análise estatística não demonstrou diferença significativa no comportamento dos dois grupos de pacientes, embora a predileção pelo terço médio tenha sido observado apenas naqueles com tumor esofágico não associado a megaesôfago. A distribuição homogênea das lesões, como observado nos pacientes do grupo 1 deste estudo, foi também referida por STREITZ et al. ${ }^{(30)}$. PINOTTI et al. ${ }^{(21)} \mathrm{e}$ BRANDALISE et al. ${ }^{(3)}$ relatam que a localização preferencial dos tumores foi o terço distal.

$\mathrm{O}$ antecedente de alcoolismo associado ao tabagismo por longo período de tempo foi referido por 29 pacientes, sendo 10 do grupo 1 e 19 do grupo 2, diferença significante $(P$ $<0,006)$. Os três pacientes desta casuística que negavam tais hábitos pertenciam ao grupo 1, levando à hipótese de que o único fator responsável pela degeneração da mucosa esofágica tenha sido a esofagite crônica, decorrente da proliferação bacteriana provocada pela estase alimentar característica do megaesôfago.

O estudo anatomopatológico dos pacientes desta série mostrou que o tumor esofágico era do tipo espinocelular em ambos os grupos. A associação do carcinoma espinocelular em paciente com megaesôfago foi relatada por vários autores ${ }^{(4,17,20)}$. $\mathrm{O}$ adenocarcinoma do esôfago pode também ser observado no seguimento de pacientes com megaesôfago submetidos a dilatação da cárdia ou miotomia da transição gastroesofágica ${ }^{(7,10,12)}$, pois tais procedimentos reduzem a pressão no esfíncter inferior do esôfago, predispondo ao refluxo gastroesofágico ${ }^{(14,15)}$. Este pode apresentar a complicação metaplasia intestinal (Barrett), lesão precursora do adenocarcinoma esofágico. O problema do câncer do esôfago no pós-operatório tardio de miotomia é enfatizado com tal veemência por CAMARA-LOPES ${ }^{(5)}$, a ponto de o autor recomendar a ressecção esofágica como única opção terapêutica para o megaesôfago, mesmo em fase não avançada da doença.

A diferenciação celular dos tumores nos pacientes com megaesôfago não diferiu daqueles do grupo $2(P=0,35)$, mostrando comportamento biológico semelhante nos dois grupos.

LAMB et al. ${ }^{(17)}$ relataram um caso de carcinoma espinocelular precoce em paciente com megaesôfago submetido a esofagectomia, com boa evolução. Infelizmente os pacientes estudados nesta casuística não tiveram o diagnóstico realizado em fase precoce, pois a maioria encontrava-se nos estádios III e IV da doença. A manifestação clínica do câncer do esôfago em geral é tardia, pois a disfagia ocorre quando a luz esofágica está severamente comprometida. Considerando que os pacientes com megaesôfago apresentam a dilatação esofágica, o diagnóstico tardio é facilmente compreensível nesta situação. A dificuldade diagnóstica é agravada nesses pacientes, pois a avaliação radiológica fica prejudicada, uma vez que ingestão de grande volume da solução baritada acoberta a lesão ${ }^{(21)}$.

O diagnóstico do tumor em fase avançada aliado à desnutrição e deficiência imunológica própria do megaesôfago ${ }^{(8)}$, restringem as opções terapêuticas cirúrgicas, levando a adoção de condutas paliativas e conseqüente sobrevida reduzida, como a observada nos pacientes desta série (Tabela 4), semelhante à relatada pela 
maioria dos autores ${ }^{(4,10,21)}$. HAAGEDOORN et al. ${ }^{(13)}$ afirmam que pacientes com tumor bem diferenciado têm, em geral, prognóstico melhor que aqueles com tumor mal diferenciado. Todavia, na presente casuística este resultado não foi observado em nenhum dos grupos estudados (Tabela 4: $P=0,36$ para o grupo 1 e $P=0,91$ para o grupo 2).

Dos 40 pacientes estudados nesta pesquisa, em apenas 1 com câncer e megaesôfago foi possível realizar a ressecção esofágica. Em nove pacientes do grupo 1 não foi indicada gastrostomia ou jejunostomia, pois a luz esofágica ampla ainda permitia razoável aporte nutricional.

Segundo SAFATLE-RIBEIRO et al. ${ }^{(28)}$, o doente com megaesôfago apresenta todos os fatores relacionados com a carcinogênose esofágica, tais como deficiências vitamínicas, decorrentes da desnutrição crônica, presença de substâncias mutagênicas na luz do órgão, resultante da proliferação bacteriana, além do alcoolismo e tabagismo, comuns nos pacientes com esta afecção.
Assim, o paciente com megaesôfago necessita de seguimento clinico e endoscópico, com vistas à detecção precoce do tumor, propiciando tratamento adequado e cura da neoplasia.

YAMAMURO et al. ${ }^{(33)}$ salientam que a endoscopia convencional não é suficiente para o seguimento desses pacientes e recomendam o uso de solução de lugol, técnica que permite a obtenção de biopsias dirigidas, em áreas não coradas, aumentando a positividade do exame e possibilitando a detecção dos tumores em fase precoce.

\section{CONCLUSÃO}

A análise dos resultados da presente casuística permite as seguintes conclusões:

O prognóstico dos pacientes com câncer do esôfago e megaesôfago é sombrio e não difere daqueles sem megaesôfago.

No paciente com megaesôfago o tumor pode se localizar em qualquer porção do órgão.

Henry MACA, Lerco MM, Oliveira WK. Esophageal cancer in patient with chagasic megaesophagus. Arq Gastroenterol. 2007;44(2):151-5.

ABSTRACT - Background - Megaesophagus constitutes a public health problem in our country since it affects individuals in the most productive phase of their lives. During the development of the disease, people suffering from it may present association with esophageal cancer. Aim - To analyze the clinical and epidemiological aspects of patients with megaesophagus and esophageal cancer. Methods - Twenty patients with megaesophagus and cancer (group 1) and 20 patients with esophageal cancer (group 2) were retrospectively analyzed. Demographic data, habits (alcoholism and smoking), tumor histological type, lesion location, cellular differentiation, staging, treatment and survival were assessed. Results - No difference was observed between the groups in relation to age, sex, lesion location, tumor histological type, cellular differentiation, staging or survival. As regards habits, the association of alcoholism with smoking was observed in a larger number of patients with esophageal cancer without the megaesophagus antecedent. Conclusions - The clinical characteristics of patients with megaesophagus and cancer do not differ from those of patients with malignant esophageal neoplasia, particularly as regards the unfavorable prognosis with the instituted treatment. Patients with megaesophagus may present esophageal tumor at any part of the organ.

HEADINGS - Esophageal achalasia. Esophageal neoplasms. Follow-up studies. 


\section{REFERÊNCIAS}

1. Barros SG, Ghisolfi ES, Luz LP, Barlem GG, Vidal RM, Wolff FH, Magno VA, Breyer HP, Dietz J, Grüber AC, Kruel CD, Prolla JC. Mate (chimarrão) é consumido em alta temperatura por população sob risco para o carcinoma epidermóide do esôfago. Arq Gastroenterol. 2000;37:25-30.

2. Brandalise NA, Leonardi LS, Terra CAD, Morisot P. Carcinoma do esôfago em megaesôfago chagásico após operação de Merendino. Rev Paul Med. 1974;83:169-72.

3. Brandalise NA, Andreollo NA, Leonardi LS, Callejas Neto F. Carcinoma associado a megaesôfago chagásico. Rev Col Bras Cir. 1985;12:196-9.

4. Brücher BL, Stein HJ, Bartels H, Feussner H, Siewert JR. Achalasia and esophageal cancer: incidence, prevalence and prognosis. World J Surg. 2001;25:745-9.

5. Camara-Lopes LH. Carcinoma of the esophagus as a complication of megaesophagus. An analysis of seven cases. Am J Dig Dis. 1961;6:742-56.

6. Chuong JJ, DuBovik S, McCallum RW. Achalasia as a risk factor for esophageal carcinoma. A reappraisal. Dig Dis Sci. 1984;29:1105-8.

7. Crema E, Lopo PN, Kalinauskas PF, Cunha GR, Silva AA. Esofagopatia chagásica associada ao adenocarcinoma do esôfago distal. Análise de 6 casos [abstract]. In: Anais do XXVI Congresso Brasileiro de Cirurgia; 2005; Rio de Janeiro, RJ. Rev Col Bras Cir. 2005;32(supl):139.

8. Crema E, Monteiro IO, Rodrigues Jr V, Silva AA. Estudo da imunossupressão dos portadores de esofagopatia chagásica por meio da avaliação das citocinas (MIG, IL-4, IL-5, IFN-G, TNF-A).[abstract]. In : Anais do XXVI Congresso Brasileiro de Cirurgia; 2005; Rio de Janeiro, RJ. Rev Col Bras Cir. 2005;32(supl):77.

9. Earlam R, Cunha-Melo JR. Oesophageal squamous cell carcinomas. A critical review of radiotherapy. Br J Surg. 1980;67:457-61.

10. Ellis FH Jr, Gibb SP, Balogh K, Schwaber JR. Esophageal achalasia and adenocarcinoma in Barrett's esophagus: a report of two cases and a review of the literature. Dis Esophagus. 1997; 10:55-60.

11. Franceschi S, Talamini R, Barra S, Baron AE, Negri E, Bidoli E, Serraino D, Vecchia C. Smoking and drinking in relation to cancers of the oral cavity, pharynx, larynx and esophagus in northern Italy. Cancer Res. 1990;50:6502-7.

12. Goodman P, Scott LD, Verani RR, Berggreen CC. Esophageal adenocarcinoma in a patient with surgically treated achalasia. Dig Dis Sci. 1990;35:1549-52.

13. Haagedoorn EM, Oldhoff J, Bender W, Clarke WD, Sleijfer DT, editores. Oncologia básica para profissionais de saúde. São Paulo: Associação Paulista de Medicina; 2000. p. 21.

14. Henry MACA, Saad LHC, Marcato PS. The importance of esophageal manometry in diagnosis and management of megaesophagus. ABCD Arq Bras Cir Dig. $1991 ; 6: 8-14$

15. Henry MACA. Megaesôfago e acalásia .In: Dani R, editor. Gastroenterologia essencial. Rio de Janeiro: Guanabara Koogan; 1998. p.96-9.

16. Katz D, Rothstein R, Schned A, Dunn J, Seaver K, Antonioli D. The development of dysplasia and adenocarcinoma during endoscopic surveillance of Barrett's esophagus. Am J Gastroenterol. 1998;93:536-41.

17. Lamb RK, Edwards CW, Pattison CW, Matheus HR. Squamous carcinoma in situ of the oesophagus in a patient with achalasia. Thorax. 1985;40:795-6.
18. Leitão AC, Braga RS. Mutagenic and genotoxic effects of mate (Ilex paraguariensis) in prokaryotic organisms. Brazilian J Med Biol Res. 1994;27:1517-25.

19. Mathews DE, Farewell VT. Using and understanding medical statistics. 3rd ed. Basel: Karger; 1996.

20. Meijssen MA, Tilanus HW, van Blankenstein M, Hop WC, Ong GL. Achalasia complicated by oesophageal squamous cell carcinoma: a prospective study in 195 patients. Gut. 1992;33:155-8.

21. Pinotti HW, Pollara WM, Gemperli R, Raia AA. O problema do câncer no megaesôfago. Rev Assoc Med Bras. 1980;26:379-81.

22. Pinotti HW, Felix VN, Domene CE, Santiago CAC, Raia A. Carcinoma do esôfago e estenose cáustica. Considerações sobre 3 casos. Rev Bras Cir. 1982;72:277-80.

23. Pintos J, Franco EL, Oliveira BV, Kowalski LP, Curado MP, Dewar R. Maté, coffee, and tea comsuption and risk of cancers of the upper aerodigestive tract in southern Brazil. Epidemiology. 1994;5:583-90.

24. Pombo L, Caramel L, Adbel A, Milezi W, Filgueiras M. Divertículo de Zenker associado a carcinoma epidermóide do esôfago. [abstract]. In: Anais do XXVI Congresso Brasileiro de Cirurgia; 2005; Rio de Janeiro, RJ. Rev Col Bras Cir. 2005;32(supl):517.

25. Prolla JC, Dietz J, Costa LA. Diferenças geográficas na mortalidade por câncer de esôfago no Rio Grande do Sul. Rev Assoc Med Bras. 1993;39:217-20.

26. Rezende JM, Lauar KM, Oliveira AR. Aspectos clínicos e radiológicos da aperistalse do esôfago. Rev Bras Gastroenterol. 1960;12:247-51.

27. Ribeiro U Jr, Posner MC, Safatle-Ribeiro AV, Reynolds JC. Risk factors for squamous cell carcinoma of the oesophagus. Br J Surg. 1997;84:281-2.

28. Safatle-Ribeiro AV, Ribeiro Jr U, Sakai P, Clarke MR, Fylyk SN, Ishioka S, GamaRodrigues JJ, Finkelstein SD, Reynolds JC. Hiperexpressão e mutação do gene p 53 em biópsias endoscópicas de pacientes com megaesôfago chagásico. GED Gastroenterol Endosc Dig. 2000;19:19-27.

29. Stein H, Feith M, Mueller J, Wermer M, Siewert JR. Limited resection for early adenocarcinoma in Barrett's esophagus. Ann Surg. 2000;232:733-42.

30. Streitz JM, Ellis FH Jr, Gibb SP, Heatley GM. Achalasia and squamous cell carcinoma of the esophagus: analysis of 241 patients. Ann Thorac Surg. 1995;59:1604-9.

31. Sugai BM, Ishioka S, Sakai P, Scabbia A, Cecconello I. Incidência de carcinoma na esofagite cáustica. GED Gastronterol Endosc Dig. 1987;6:91-4.

32. Victora CG, Muñoz N, Horta BL, Ramos EO. Patterns of maté drinking in a Brazilian City. Cancer Res. 1990;50:7112-5.

33. Yamamuro EM, Cecconello I, Iriya K, Tomishigue T, Oliveira MA, Pinotti HW. Lugo dye endoscopy for analysis of esophageal mucosa in achalasia. Hepatogastroenterology. 1999;46:1687-91.

34. Zilberstein B. Esofagectomia cérvico abdominal por via transdiafragmática no tratamento cirúrgico do câncer do esôfago: resultados e evolução a longo prazo [tese]. São Paulo: Faculdade de Medicina da Universidade de São Paulo; 1988. 